\title{
A Pilot Study of Health Coaching on Older Adults' Personal Healthcare and Maintenance during the Outbreak of Covid-19 in Singapore
}

\author{
Yuanyuan $\mathrm{Cao}^{1 *}$, Yuezhong Liu², Joshua Ho Yin Lai ${ }^{3}$, Yin-Leng Theng \\ ${ }^{1}$ Centre for Healthy and Sustainable Cities (CHESS), Wee Kim Wee School of Communication \& Information, Nanyang \\ Technological University, Singapore \\ ${ }^{2}$ Ageing Research Institute for Society and Education (ARISE), Nanyang Technological University, Singapore \\ ${ }^{3}$ School of Social Sciences, Nanyang Technological University, Singapore \\ Email: *yycao@ntu.edu.sg
}

How to cite this paper: Cao, Y.Y., Liu, Y.Z., Lai, J.G.Y. and Theng, Y.-L. (2021) A Pilot Study of Health Coaching on Older Adults' Personal Healthcare and Maintenance during the Outbreak of Covid-19 in Singapore. Health, 13, 165-177.

https://doi.org/10.4236/health.2021.132015

Received: January 12, 2021

Accepted: February 23, 2021

Published: February 26, 2021

Copyright $\odot 2021$ by author(s) and Scientific Research Publishing Inc. This work is licensed under the Creative Commons Attribution International License (CC BY 4.0).

http://creativecommons.org/licenses/by/4.0/

\begin{abstract}
With a rapidly ageing population in Singapore, older adults and their family members face challenges of age-related diseases, caregiver stress, and increasing demand for caregiving services. To address this pressing issue, this pilot study aims to introduce online health coaching for older adults in Singapore and evaluate its effectiveness on maintenance of personal healthcare. In the midst of the COVID-19 outbreak, older adults' activities were largely restricted by measures on social distancing. The online health coaching provided a solution for older adults to achieve a healthy lifestyle in a contactless manner. In this pilot study, 18 older adults ( $\geq 55$ years old) who were willing to be coached by trained health coaches $(\mathrm{n}=10)$ for 8 weeks (once a week) were recruited. Pre- and post-surveys with older adults were conducted. Furthermore, health coaching booklet was used to control the quality of coaching and to record diet and exercise plans. The pilot study demonstrated that the health coaching provided older adults with knowledge of nutrition and exercise, raised their awareness of well-being in terms of daily meals and regular exercise, and provided an alternative to maintain a healthy lifestyle amidst global pandemic. Additionally, we also identified that the older adults' satisfactions with health coaching were positively associated with educational levels $\left(\mathrm{R}^{2}=0.31\right)$. Lastly, this pilot study highlighted that health coaching standardization process for older adults is critical for researchers and healthcare practitioners in the future.
\end{abstract}

\section{Keywords}

Health Coaching, Personal Health Care, Health Maintenance, Quality of Life 


\section{Introduction}

Health coaching is defined as assisting patients to gain knowledge, skills, tools and confidence to become active participants in their personal care to achieve self-identified goals [1]. It intends to encourage and educate individuals to develop good health-maintaining, lifestyle habits, and improve their overall physical and mental well-being by building a collaborative relationship between a health coach and a client [2]. Health coaches act as facilitators and companions to remind their clients to follow proper exercise, diet, and medication plans [3]. Compared to healthcare professionals that may differ between visits, health coaches are tagged to the patients thus they are able to build rapport and develop a deeper understanding of the patient's needs [4]. In addition to focusing on physical health, health coaching also emphasizes individuals' mental health by offering emotional support through scheduled meetings, thereby allowing patients to comprehensively improve their overall wellbeing [5].

Previous studies showed a need for health practitioners to formulate intervention programme for patients [6] and also identified key motivations of introducing health coaches into the healthcare system. The primary motivations included 1) the inability of primary care physicians to meet the need of every patient, 2) the lack of patient understanding of physician instructions, 3) the lack of patient participation in shared decision-making, and 4) lower rates of medication adherence and lifestyle changes [7] [8] [9] [10].

A study by Cinar and Schou [11] investigated the effectiveness of health coaching in improving oral health and diabetic outcomes of its clients. Results indicated that over a span of 16 months, patients in the health coaching condition experienced marked improvements in diabetic outcomes, a significant reduction in fasting blood glucose levels, compared to patients in the control condition. Additionally, periodontal health of patients, secondary objective, also improved considerably under the health coaching programme [11]. Similarly, the study by Wolever et al. [12] corroborated the above findings by demonstrating the effectiveness of health coaching programmes in improving health-related outcomes of diabetic participants. This study indicated that, within a span of 6 months, participants $(n=30)$ in the health coaching condition are significantly more likely to adhere to regular medication plans and reported having significant improvements in their perceptions of diabetes than participants in the control group $(n=26)$. Furthermore, participants in health coaching condition also experienced a significant reduction in stress and improvement in the quality of life [12]. These findings demonstrated the effectiveness of health coaching by improving their health-related outcomes and instilling health maintaining regimes in their daily lives.

Singapore is a rapidly ageing society, with an average life expectancy of 78 years amongst its citizens. It has projected that the number of Singaporeans aged 65 and above will double to 900,000 by 2030 , which means that one in four Singaporeans will fall into this age group by then [13]. With the progressively grey- 
ing population, chronic diseases become an increasingly prominent societal concern [14]. To tackle chronic diseases effectively, patients need to religiously adhere to the medication plan and health-related lifestyle changes [15]. Unfortunately, occasional clinical visitations to primary healthcare providers do not guarantee an improvement in chronic diseases as findings suggest that behaviours of patients generally do not change across the limited professional consultations sessions [15]. Compounded with the central healthcare philosophy in Singapore for individuals to be financially self-reliant [13], frequent visits to healthcare providers are not an economically viable and ideal option. Therefore, health coaching serves as a bridge between the professional advice of medical practitioners and the concrete actions needed to improve one's health-related outcomes.

This pilot study aimed to investigate the feasibility of online health coaching in Singapore during the outbreak of COVID-19, so as to provide an alternative way to facilitate older adults to personal health care and maintenance. In this study, we focused specifically on exercise and diet planning with the aid of online health coaches. Hence, this study explored the following research questions (RQs):

1) What benefits (physical and psychosocial) can online health coaching provide to older adults in Singapore?

2) What are the factors that influence older adults' satisfaction towards online health coaching programme?

3) How can current health coaching programme be improved to better serve future clients in Singapore?

\section{Study Design}

\subsection{Participants}

The inclusion criteria for the recruitment of older adults for the intervention of online health coaching and pre and post-surveys were that they were 1) aged 55 years and above; 2 ) had voluntarily consented to take part in the study; 3 ) were able to communicate and express themselves clearly (to ensure data collection in the form of a survey); 4) had willingness of maintaining or enhancing health status. Initially, the recruitment faced various challenges due to the 2020 Singapore circuit breaker (CB) measures. Instead of recruiting within Senior Activity Centers, we had to circulate the advertisement of recruitment in volunteer groups through WhatsApp. For the recruitment of health coaches, we approached the contact person in Regional Health System Office of National University Health System. Through her kind assistance and coordination, several interested parties volunteered as health coaches to serve the older adults. Older adults participants $(n=18$; Female $=16$, Male $=2)$ were recruited via convenience sampling to take part in this pilot study during March and April 2020. The mean age of participants was 64.4 years $(\mathrm{SD}=7.31$ ). Amongst the participants, 15 were Chinese, two were Indians, and one was Burmese. Education levels and 
self-reported health status varied among participants as well. 15 of them received education higher than secondary school. Detailed demographic information can be found in Table 1 .

\subsection{Method}

Prior to the online health coaching intervention, training, consisted of four modules (introduction to gerontology, nutrition for older adults, exercise and

Table 1. Demographic information of participants (older adults) $(n=18)$.

\begin{tabular}{|c|c|}
\hline Demographic Characteristic & Total $(n=18)$ \\
\hline Age $\left(S D^{*}\right)$ & $64.6(7.31)$ \\
\hline \multicolumn{2}{|l|}{ Gender } \\
\hline Male & 2 \\
\hline Female & 16 \\
\hline \multicolumn{2}{|l|}{ Race } \\
\hline Chinese & 15 \\
\hline Indian & 2 \\
\hline Other (Burmese) & 1 \\
\hline \multicolumn{2}{|l|}{ Education Level } \\
\hline Primary School & 3 \\
\hline Secondary School & 9 \\
\hline Junior College/Polytechnic & 1 \\
\hline University & 5 \\
\hline \multicolumn{2}{|l|}{ Presence of Family Support } \\
\hline Yes & 3 \\
\hline No & 15 \\
\hline \multicolumn{2}{|l|}{ Self-reported Health Status (Pre-test) } \\
\hline Very poor & 0 \\
\hline Poor & 0 \\
\hline Average & 8 \\
\hline Good & 7 \\
\hline Very Good & 3 \\
\hline \multicolumn{2}{|c|}{ Self-reported Clinical Characteristics } \\
\hline Asthma & 2 \\
\hline Hypertension & 2 \\
\hline Obesity & 1 \\
\hline Type-2 Diabetes & 2 \\
\hline Hypercholesterolemia & 4 \\
\hline
\end{tabular}

${ }^{*}$ SD: standard deviation. 
fitness, and health coaching basics), was provided to health peers from the $\mathrm{Na}$ tional University Health System (NUHS) and volunteers from Tzu Chi Foundation (TCF) at the end of May 2020. In total, we had 10 health coaches trained by professional trainers. Additionally, a health coaching booklet was designed to guide health coaches to follow the standardized protocol during coaching. Table 2 shows the summarized content of the health coaching booklet. It facilitated the process of delivering the knowledge regarding nutrition and exercise as well as controlling the quality of health coaching.

The period of the health coaching intervention was 2 months, with weekly sessions. Eight sessions were provided by health coaches between June and September 2020. To avoid any risk infection of Covid-19, all sessions were conducted online via Zoom. One health coach will serve one older adult throughout eight sessions. Since we had fewer numbers of health coaches $(n=10)$ than older adults $(\mathrm{n}=18)$, we intentionally conducted health coaching in two batches. Matches between health coaches and older adults were made according to older adults' preferences (gender, religion, age etc.). After pairing them, the first sessions were scheduled based on their availability. A dedicated student assistant was assigned to a pair of health coaches and their assigned older adults. The student assistant played the role as coordinator to notify participants of the upcoming sessions, make appointments with participants and share screen with participants if health coaches need to show older adults the "My Healthy Plate" or "7 Easy Exercises". The duration of each session was between 30 minutes to an hour. Figure 1 shows how the eight sessions were designed and conducted.

A mixed-method approach was applied in this study using both qualitative and quantitative methods, comprising health coaching booklets recorded during each session by health coaches and pre- and post-surveys done by older adults. Measurements included SF-12 Health Survey, UCLA Loneliness Scale, Medical Outcomes Study Social Support Survey, Adherence to Refills and Medications Scale, Health-related quality of life, and Geriatric Depression Scale [16]-[21].

Table 2. Summarized content of health coaching booklet

\begin{tabular}{|c|c|}
\hline Category & Example \\
\hline $\begin{array}{l}\text { Guide for Health } \\
\text { Coaching }\end{array}$ & Motivational Interviewing (OARS) \\
\hline $\begin{array}{l}\text { Guide for Healthy } \\
\text { Eating }\end{array}$ & My Healthy Plate (recommended by Health Promotion Board, Singapore) \\
\hline \multirow[t]{2}{*}{ Guide for Exercise } & 7 Easy Exercises (recommended by Health Promotion Board, Singapore) \\
\hline & Week 1 (Session 1): S.M.A.R.T. Goal setting \\
\hline $\begin{array}{l}\text { Guide for } \\
\text { Coaching Sessions }\end{array}$ & $\begin{array}{l}\text { Week } 2 \text { - Week } 7 \text { (Session } 2 \text { - Session 7): } \\
\text { follow up with the initial goal: "how much have you achieved your goal in diet } \\
\text { plan/exercise plan?", "what were difficulties of achieving your goa?" }\end{array}$ \\
\hline & $\begin{array}{c}\text { Week } 8 \text { (Session } 8 \text { ): review the initial goal, satisfaction towards health coaching } \\
\text { by older adults }\end{array}$ \\
\hline
\end{tabular}




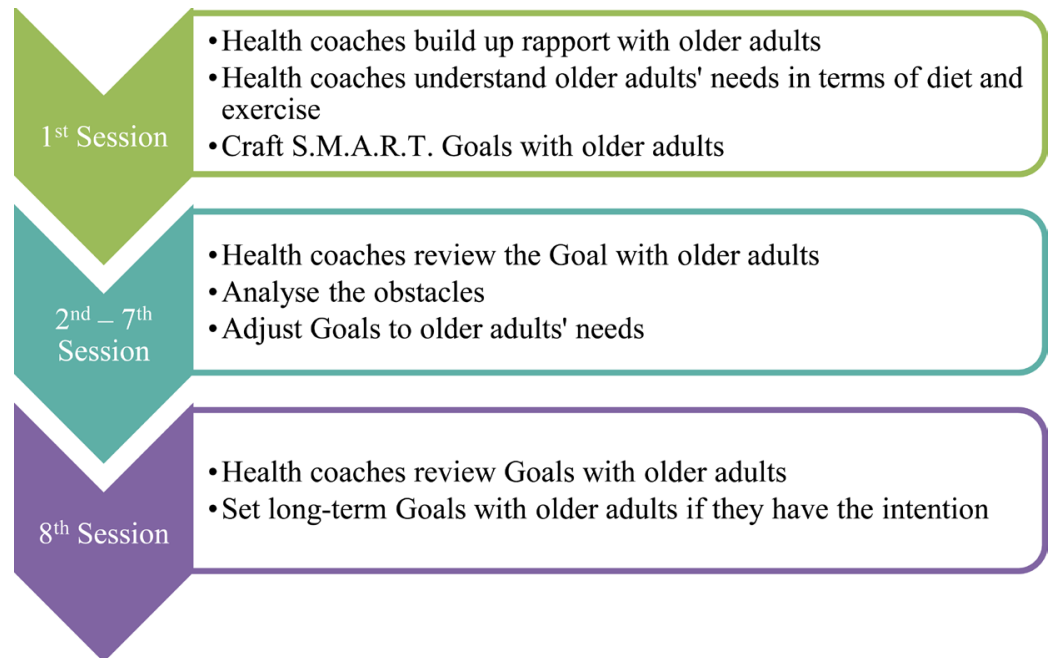

Figure 1. Design of online health coaching process.

\section{Results}

\subsection{Descriptive Statistics}

The descriptive statistics for the measures used in our study are summarised in Table 3, Table 4 and Figure 2 \& Figure 3.

Based on the high averaged means observed in the contentment of health-coaching scale (mean $=4.19$, maximum $=5$ ), it was evident that participants generally enjoyed the health coaching programme. Similarly, a high averaged means score was also observed in the satisfaction with the online health coaching scale (mean $=3.35$, maximum $=4$ ), which suggested that participants' expectations of the programme were met and they received good care in the programme. In combination, these high scores illustrated the potential of the current health coaching programme in delivering the mentor-client health-oriented service. More importantly, it demonstrated that older adults in Singapore are receptive to health coaching programmes.

Table 3. Descriptive statistics of scales.

\begin{tabular}{ccc}
\hline Measurement & Mean & Standard Deviation (SD) \\
\hline Contentment of health-coaching & 4.19 & 0.74 \\
Satisfaction with health-coaching & 3.35 & 0.47 \\
SF-12 health scale (physical health pre) & 47.16 & 6.28 \\
SF-12 health scale (physical health post) & 48.85 & 8.27 \\
SF-12 health scale (mental health pre) & 53.61 & 6.44 \\
SF-12 health scale (mental health post) & 56.88 & 4.16 \\
\hline
\end{tabular}

Table 4. Quality of life (Level of health).

\begin{tabular}{ccc}
\hline Measurement (mean, $S D)$ & Pre-test & Post-test \\
\hline Level of health & $79.39(11.93)$ & $84.28(10.37)$ \\
\hline
\end{tabular}




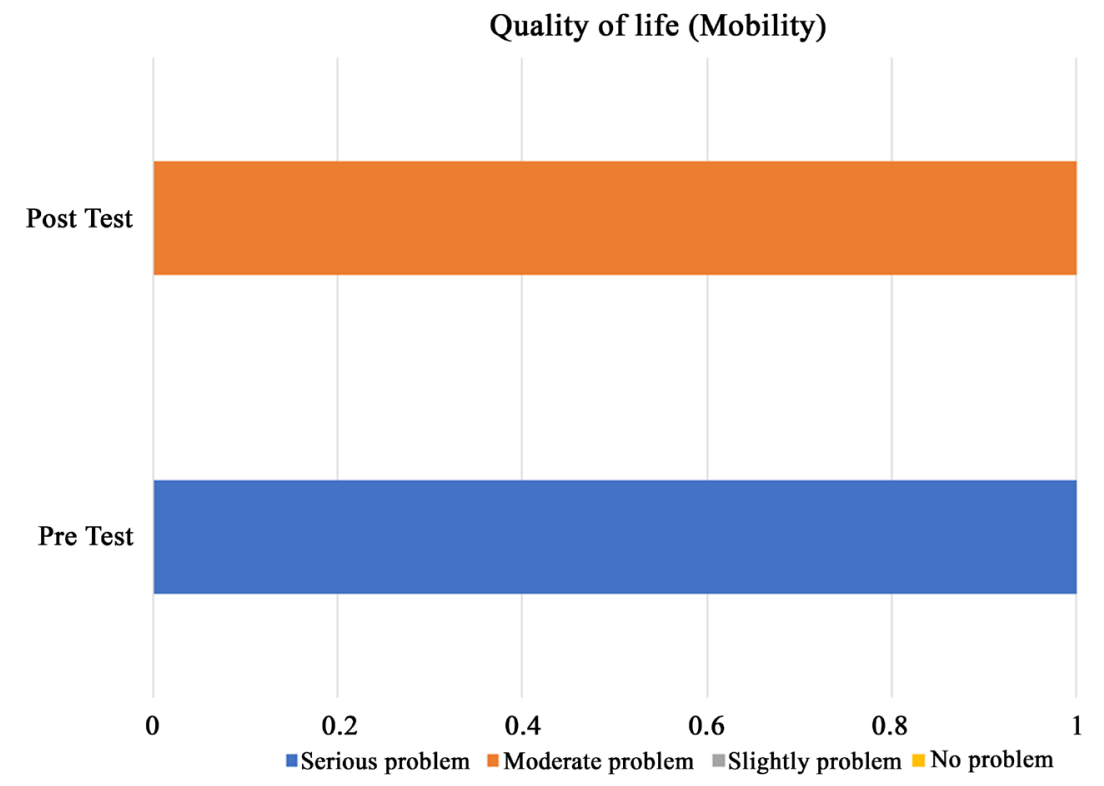

Figure 2. Quality of life (mobility).

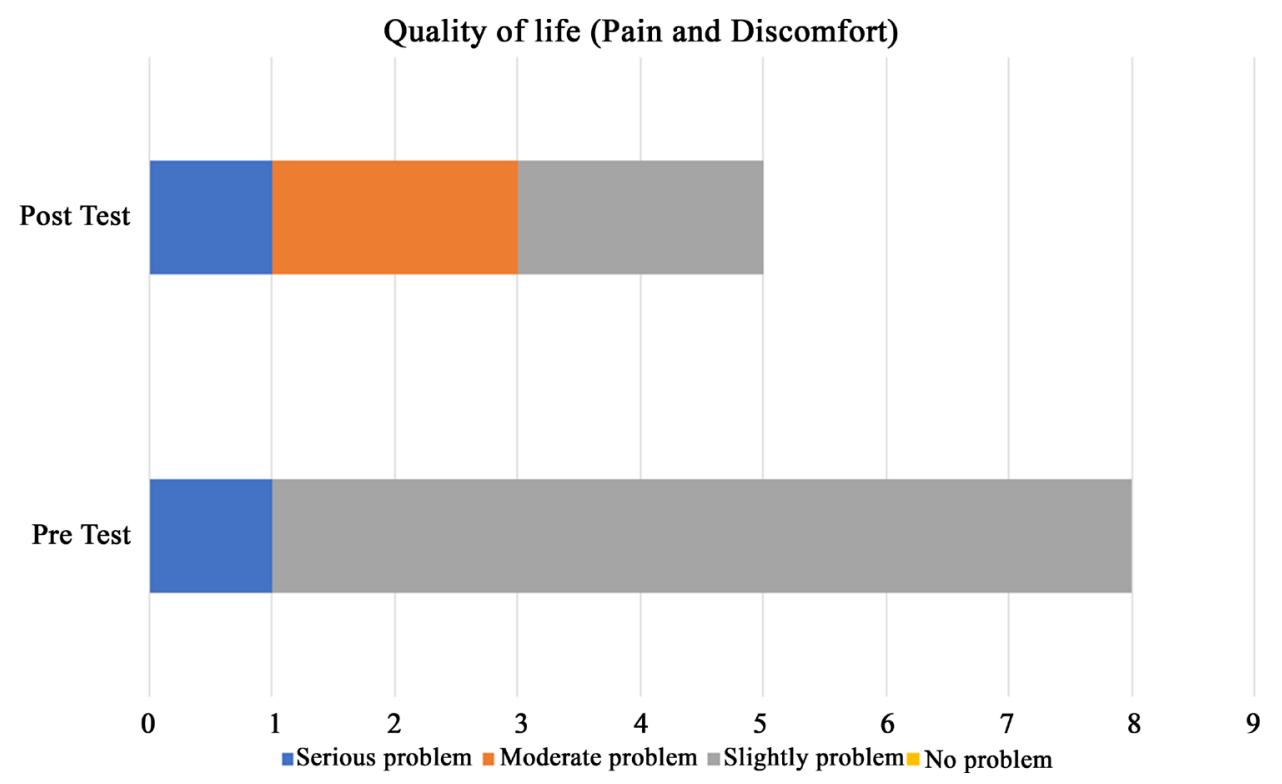

Figure 3. Pain and discomfort.

The quality of life scale (EQ-5D) had a total of 6 items and aimed to measure 5 dimensions of a participant's health, namely mobility, self-care, usual activities, pain and discomfort, and anxiety. Each item corresponded to one domain, with the last item measuring the general health status of the participants [15].

Based on Table 4, the self-reported levels of health experienced by participants showed an improvement from 79.39 during pre-test to 84.28 during post-test. Although the improvement was not statistically significant, the results demonstrated promise in the beneficial effects of the health coaching programme.

With regard to mobility, as shown in Figure 2, one participant reported having serious problems walking during the pre-test. At the end of the health 
coaching sessions, the same participant demonstrated mild improvements in mobility as he reported having lesser problems walking compared to pre-health coaching. However, it was uncertain that the improvement was due to the outcome of the health coaching.

With regard to pain and discomfort, the results are mixed (Figure 3). Most participants $(n=5)$ experienced an improvement in the degree of pain after the health coaching sessions. On the other hand, some participants $(n=3)$ reported having an increase in the intensity of pain after the health coaching sessions (Table 5).

\subsection{Regression Analysis}

The assumptions of regression include:

1) Independence of Observations assumed for regression analysis;

2) Normality looking at the normal probability plot generated between the independent variable, IV (education levels) and DV (satisfaction with health coaching), the residuals of the regression neatly aligning with the normality line thus normality assumption is satisfied;

3) Homoscedasticity referring to whether residuals are evenly distributed across all levels of the dependent variable. Looking at the scatter-plot generated between the independent variable, education level, and DV, the assumption of homoscedasticity appears to be violated. Nonetheless, the research team proceeded with the regression analysis as the violation of homoscedasticity is likely due to our small sample size.

From the regression analysis, with education levels of participants (Table 1) as independent variable and satisfaction of health coaching as the dependent variable, we found the following results:

1) Education level was a significant predictor of health-coaching satisfaction, $\mathrm{R}^{2}=0.31, \mathrm{~F}(1,16)=7.23, \mathrm{p}=0.016<0.05$. Specifically, the higher the education levels of the participant, the more likely he or she was satisfied with the health coaching programme.

Table 5. Changes in pain and discomfort of participants.

\begin{tabular}{ccc}
\hline Participant & Pre Test (Pain and discomfort) & Post Test (Pain and discomfort) \\
\hline 1 & Serious problems & Moderate problems \\
2 & Slight problems & Moderate problems \\
3 & Slight problems & No problems \\
4 & Sight problems & Slight problems \\
5 & Slight problems & No problems \\
6 & Slight problems & Serious problems \\
7 & Slight problems & No problems \\
8 & No problems & Slight problems \\
9 & Slight problems & No problems \\
\hline
\end{tabular}


2) Regressions for other demographic variables, gender and races, were not ran as they were not key variables of interest and the groups within the variables were heavily unbalanced.

\subsection{Qualitative Analysis}

During the circumstances of Covid-19, older adults' activities were largely restricted by measures on social distancing and cancellation of various social activities. The online health coaching provided a solution for older adults to achieve a healthy lifestyle in a timely and contactless manner. Most of them provided the highest score (5/5) for their health coaches and this programme at the last session, which showed their satisfaction towards the programme. They also admitted that health coaching facilitated them in forming good eating and exercising habits throughout the two months. Although it was novel to all of them, they found that it was beneficial to them in the following aspects:

1) They felt that the biggest change brought by this programme was that it raised their awareness of well-being in terms of daily meals and regular exercise. They overlooked this aspect of life as they did not realize the causal relationships between their current behaviors and the consequences in the future.

2) They might have overlooked the crucial things in their lives amidst the chaos brought by fear of Covid-19. The health coaching, at large, provided the opportunity to be conscious about their own lives and take personal care of themselves. They shared that "it feels so good to have a coach who is equipped with skills and knowledge to teach me something I have overlooked in daily life", and "it has been a long time since I really thought about what I should eat for the next meal seriously".

3) The companionship of health coaches made older adults felt comforted, especially when they were forced to stop community activities (e.g. morning exercises at Senior Activity Centres) during Covid-19 outbreak. In general, they felt less lonely. For some of them, they realized that there was someone, other than doctors and nurses, that could talk to them on topics of health.

It has been also found that when older adults were self-motivated in recording their meals, planning meals, and communicating with health coaches about their dietary plans. Additionally, they were also generally able to achieve the initial goals they set and expressed feeling more confidently in achieving goals in the future. From conversations with health coaches after the eight sessions, they shared that they could be motivated by older adults as well when they were told that health coaching was helpful. Health coaches played the role as partners and companions of older adults, while older adults could also encourage health coaches to apply these health advices to their daily life as well.

Regarding the improvement needed in the online health coaching programme, older adults shared that they had built rapport between them and health coaches throughout two months, and they would like to be coached for longer period. Additionally, the content of coaching could be customized ac- 
cording to different personal health care needs. Client-centred approach could be investigated to meet further needs on health coaching.

\section{Discussion}

This pilot study aimed to study the feasibility of the online health coaching using a tightly designed online coaching programme while providing a standardized booklet in the coaching process. Healthcare services called for the standardization of health coaching and concluded that uniformed training and credentialing are imperative to define the scope of practice for the healthcare profession. Such standardization and the research process are essential to understanding best practices and identifying the scope of health coaching practice. If this proposed online coaching were to be accepted into the existing healthcare system, it should not only be considered as a valuable online intervention approach but also a suggestion on healthcare policy making. Hence, health coaches should have not only a common core of health knowledge but also a similar set of effective, evidence-based intervention strategies.

This study attempted to address three research questions with the analyses:

RQ1: Benefits (physical and psychosocial) that health-coaching programme offers for its participants.

From the results, we identified that older adults showed great interest in coaching and provided positive feedback to this programme and health coaches. This health-coaching programme helped old adults to be aware of their wellbeing in terms of daily meals and regular exercise. With the companionship of health coaches, this programme made older adults feel comforted, especially when they were forced to stop community activities (e.g. morning exercise at a Senior Activity Centre) during the Covid-19 outbreak.

RQ2: Factors that influenced the satisfaction of the participants towards the online health coaching programme.

As this study was a pioneering pilot study, these patterns of improvements were promising and showed that health coaching could improve health-related outcomes for old adults in Singapore. Based on the regression analysis, education level was a significant predictor of health-coaching satisfaction $\left(\mathrm{R}^{2}=0.31\right)$. Specifically, the higher the education level of the participant, the more likely he or she was satisfied with the health coaching programme. Other demographic variables, gender and races, were not key predictors of interest and the groups within the variables were heavily unbalanced thus the authors excluded the statistical analysis of these variables.

RQ3: Improvement of health coaching needed to better serve future clients in Singapore.

In general, older adults shared positive feedback with us on the quality of health coaching and potential of implementation of this programme to a large group of participants. Meanwhile, older adults shared the good relationship built between them and health coaches, therefore, the expectation of longer period of 
health coaching. It has been an integral part of their life after the two-month intervention. Additionally, there is a request to customize the content of coaching according to differing needs in personal health care. Client-centered approach could be investigated to adapt to the future of health coaching for older adults in Singapore.

\section{Conclusion}

This study introduced the role of health coaches to provide online health coaching sessions for older adults who needed to monitor their health and maintain a healthy lifestyle in a timely and contactless manner. We conducted this pilot study and recruited 18 older adults ( $\geq 55$ years old) to be coached by trained health coaches $(\mathrm{n}=10)$ for 8 weeks (once a week). Pre- and post-survey were conducted before the first coaching session and after the last coaching session, respectively. During each session, the standard protocol was used to control the quality of coaching and to track changes in exercise and nutrition for older adults. It demonstrated that online health coaching provided an alternative solution for older adults to achieve a healthy and balanced lifestyle in a timely and contactless manner during the circumstances of Covid-19, and raised their awareness of wellbeing in terms of daily meals and regular exercise.

There were constraints faced in the study. These difficulties needed to be addressed when conducting online health coaching research, and that included monitoring the daily physical activity, emotion, and varying participants' medical conditions. The limitations also included study design issues and getting high-quality data from both qualitative and quantitative approaches that could be accepted by the scientific and medical communities.

Future studies of health coaching are needed to examine strategies of the coaching process, for example, we need to standardize the health coaching process (uniformed training and credentialing) for older adults. Furthermore, it is important to identify the best motivational interviewing technique to improve older adults' health-related outcomes. As the ageing population is heterogeneous, we also need to consider determinants of an appropriate health coach, for example, based on participants' profiling, it is essential to customize components of the coaching process to match older adults' characteristics. Other requirements worth considering include the duration and frequency of coaching session which will influence the adherence to lifestyle changes or other outcomes. Moreover, to define the scope of practice and effectiveness for the coaching profession, future health coaching investigations also need to specify the aspects of coaching such as targeting specific lifestyle chronic disease (e.g., Type 2 Diabetes Mellitus, hypertension) or improving certain dimensions of qualities of life (e.g., living independence, happiness). In summary, a client-centered health coaching standardization process (uniformed training and credentialing) for older adults is critical to study the effectiveness of the online health coaching programme. 


\section{Acknowledgements}

The authors would like to thank ARISE Strategic Initiatives Funding 2017 (Project Ref. No.: ARISE/2017/19) for supporting this study.

\section{Conflicts of Interest}

The authors declare no conflicts of interest regarding the publication of this paper.

\section{References}

[1] Bennett, H.D., Coleman, E.A., Parry, C., Bodenheimer, T. and Chen, E.H. (2010) Health Coaching for Patients with Chronic Illness. Family Practice Management, 17, 24-29.

[2] Huffman, M. (2007) Health Coaching: A New and Exciting Technique to Enhance Patient Self-Management and Improve Outcomes. Home Healthcare Nurse, 25, 271-276. https://doi.org/10.1097/01.NHH.0000267287.84952.8f

[3] Palmer, S., Tubbs, I. and Whybrow, A. (2003) Health Coaching to Facilitate the Promotion of Healthy Behaviour and Achievement of Health-Related Goals. International Journal of Health Promotion and Education, 41, 91-93. https://doi.org/10.1080/14635240.2003.10806231

[4] Naik, A.D., et al. (2012) Behavioral Health Coaching for Rural-Living Older Adults with Diabetes and Depression: An Open Pilot of the HOPE Study. BMC Geriatrics, 12, 37. https://doi.org/10.1186/1471-2318-12-37

[5] Sherifali, D. (2017) Diabetes Coaching for Individuals with Type 2 Diabetes: A State-of-the-Science Review and Rationale for a Coaching Model. Journal of Diabetes, 9, 547-554. https://doi.org/10.1111/1753-0407.12528

[6] Devlin, N.J., Shah, K.K., Feng, Y., Mulhern, B. and van Hout, B. (2018) Valuing Health-Related Quality of Life: An EQ-5D-5L Value Set for England. Health Economics, 27, 7-22. https://doi.org/10.1002/hec.3564

[7] Bodenheimer, T. (2007) A 63-Year-Old Man with Multiple Cardiovascular Risk Factors and Poor Adherence to Treatment Plans. JAMA, 298, 2048-2055.

https://doi.org/10.1001/jama.298.16.jrr70000

[8] Braddock, C.H., Edwards, K.A., Hasenberg, N.M., Laidley, T.L. and Levinson, W. (1999) Informed Decision Making in Outpatient Practice: Time to Get Back to Basics. JAMA, 282, 2313-2320. https://doi.org/10.1001/jama.282.24.2313

[9] Haynes, R.B., McDonald, H.P. and Garg, A.X. (2002) Helping Patients Follow Prescribed Treatment: Clinical Applications. JAMA, 288, 2880-2883. https://doi.org/10.1001/jama.288.22.2880

[10] Østbye, T., et al. (2005) Is There Time for Management of Patients with Chronic Diseases in Primary Care? Annals of Family Medicine, 3, 209-214.

https://doi.org/10.1370/afm.310

[11] Basak Cinar, A. and Schou, L. (2014) Health Promotion for Patients with Diabetes: Health Coaching or Formal Health Education? International Dental Journal, 64, 20-28. https://doi.org/10.1111/idj.12058

[12] Wolever, R.Q., et al. (2010) Integrative Health Coaching for Patients with Type 2 Diabetes: A Randomized Clinical Trial. Diabetes Education, 36, 629-639. https://doi.org/10.1177/0145721710371523

[13] Ministry of Health (2016) I Feel Young in My Singapore: Action Plan for Successful 
Ageing.

https://www.moh.gov.sg/docs/librariesprovider3/action-plan/action-plan.pdf

[14] Tracy, R.P. (2003) Emerging Relationships of Inflammation, Cardiovascular Disease and Chronic Diseases of Aging. International Journal of Obesity and Related Metabolic Disorders. Journal of the International Association for the Study of Obesity, 27, S29-S34. https://doi.org/10.1038/sj.ijo.0802497

[15] Wong-Rieger, D. and Rieger, F.P. (2013) Health Coaching in Diabetes: Empowering Patients to Self-Manage. Canadian Journal of Diabetes, 37, 41-44. https://doi.org/10.1016/j.jcjd.2013.01.001

[16] Turner-Bowker, D. and Hogue, S.J. (2014) Short Form 12 Health Survey (SF-12) BT. In: Michalos, A.C., Ed., Encyclopedia of Quality of Life and Well-Being Research, Springer, Berlin, 5954-5957. https://doi.org/10.1007/978-94-007-0753-5 2698

[17] Russell, D.W. (1996) UCLA Loneliness Scale (Version 3): Reliability, Validity, and Factor Structure. Journal of Personality Assessment, 66, 20-40. https://doi.org/10.1207/s15327752jpa6601 2

[18] Schulz, U. and Schwarzer, R. (2003) Soziale Unterstützung bei der Krankheitsbewältigung: Die Berliner Social Support Skalen (BSSS) [Social Support in Coping with Illness: The Berlin Social Support Scales (BSSS)]. Diagnostica, 49, 73-82. https://doi.org/10.1026//0012-1924.49.2.73

[19] Kripalani, S., Risser, J., Gatti, M.E. and Jacobson, T.A. (2009) Development and Evaluation of the Adherence to Refills and Medications Scale (ARMS) among Low-Literacy Patients with Chronic Disease. Value in Health, 12, 118-123. https://doi.org/10.1111/j.1524-4733.2008.00400.x

[20] Herdman, M., et al. (2011) Development and Preliminary Testing of the New Five-Level Version of EQ-5D (EQ-5D-5L). Quality of Life Research, 20, 1727-1736. https://doi.org/10.1007/s11136-011-9903-X

[21] Yesavage, J.A. and Sheikh, J.I. (1986) 9/Geriatric Depression Scale (GDS). Clinical Gerontologist, 5, 165-173. https://doi.org/10.1300/J018v05n01 09 